
\title{
$\angle$ Research Square \\ Polygenic association with severity and long-term outcome in eating disorder cases
}

\section{Therese Johansson}

Uppsala University https://orcid.org/0000-0003-1043-7065

\section{Andreas Birgegård}

Uppsala University

\section{Ruyue Zhang}

Karolinska Institute https://orcid.org/0000-0001-5747-9428

\section{Sarah Bergen}

Karolinska Institutet

Mikael Landén

Uppsala University

Liselotte Petersen

Aarhus University

Cynthia Bulik

Uppsala University

Christopher Hübel ( $\nabla$ christopher.huebel@ki.se )

Karolinska Institutet https://orcid.org/0000-0002-1267-8287

\section{Article}

Keywords: anorexia nervosa (AN), SEED, Clinical Impairment Assessment (CIA), body mass index (BMI)

Posted Date: October 7th, 2021

DOI: https://doi.org/10.21203/rs.3.rs-724835/v1

License: (c) (1) This work is licensed under a Creative Commons Attribution 4.0 International License.

Read Full License

Version of Record: A version of this preprint was published at Translational Psychiatry on February 16th, 2022. See the published version at https://doi.org/10.1038/s41398-022-01831-2. 


\section{Abstract}

About $20 \%$ of individuals with anorexia nervosa (AN) remain chronically ill. Therefore, early identification of poor outcome could improve care. Genetic research has identified regions of the genome associated with AN. Patients with anorexia nervosa were identified via the Swedish eating disorder quality registers Stepwise and Riksät and invited to participate in the Anorexia Nervosa Genetics Initiative. First, we associated genetic information longitudinally with eating disorder severity indexed by scores on the Clinical Impairment Assessment (CIA) in 2,843 patients with lifetime AN with or without diagnostic migration to other forms of eating disorders followed for up to 16 years (mean $=5.3 \mathrm{yrs}$ ). Second, we indexed development of a severe and enduring eating disorder (SEED) by a high CIA score plus a followup time $\geq 5$ years. We associated individual polygenic scores (PGSs) indexing polygenic liability for AN, schizophrenia, and body mass index (BMI) with severity and SEED. After multiple testing correction, only the BMI PGS when calculated with traditional clumping and $\mathrm{p}$ value thresholding was robustly associated with disorder severity ( $\beta P G S=1.30 ; 95 \% \mathrm{Cl}: 0.72,1.88 ; p=1.2 \times 10-5)$ across all $p$ value thresholds at which we generated the PGS. However, using the alternative PGS calculation method PRS-CS yielded inconsistent results for all PGS. The positive association stands in contrast to the negative genetic correlation between BMI and AN. Larger discovery GWASs to calculate PGS will increase power, and it is essential to increase sample sizes of the AN GWASs to generate clinically meaningful PGS as adjunct risk prediction variables. Nevertheless, this study provides the first evidence of potential clinical utility of PGSs for eating disorders.

\section{Introduction}

Eating disorders are complex psychiatric conditions that arise from a combination of genetic and environmental factors. ${ }^{1}$ Anorexia nervosa (AN) is among the most serious and deadly of all psychiatric disorders ${ }^{2}$ as only $30 \%$ of patients achieve remission ${ }^{3}$ and $20 \%$ remain chronically ill. ${ }^{4}$ A severe and enduring eating disorder (SEED) describes those with AN or bulimia nervosa (BN) with chronic symptoms, treatment non-response, ${ }^{5-7}$ and long duration of illness, variably defined as five, ${ }^{8}$ six $^{9}$ seven, $^{10,11}$ or ten ${ }^{12}$ years.

In AN, concurrent anxiety or depressive symptoms, psychosocial difficulties, ${ }^{13}$ long duration of illness prior to hospitalization, low $\mathrm{BMI}$, and inadequate weight gain during hospitalization are associated with poor outcome in general and 21 years after initial hospitalization. ${ }^{14,15}$ In $\mathrm{BN}$, findings are mixed with a high frequency of compensatory behaviours ${ }^{16}$ and comorbid psychiatric diagnoses associated with poor outcome, ${ }^{17-19}$ whereas perfectionism, obsessionality, anxiety, and genetic factors may increase the likelihood of developing a SEED. ${ }^{20}$ Studies to identify predictors of poor outcome for binge-eating disorder (BED) have not had adequate statistical power. ${ }^{21,22}$

The largest genome-wide association study (GWAS) published as of 2020 associated eight risk loci with AN and indicated a genetic sharing between AN and BMI $\left(r_{\mathrm{g}}=-0.32\right)$ and between AN and schizophrenia 
$\left(\mathrm{SCZ} ; r_{\mathrm{g}}=0.25\right)$, corroborating observed comorbidity and familiality ${ }^{23}$ in clinical and epidemiological studies. ${ }^{24-26}$ These findings implicate both anthropometric and psychiatric factors in the origin of AN. ${ }^{27}$ Individual genetic liability to, for example, high BMI can be expressed as polygenic scores (PGS). PGSs are calculated for each individual by weighting the genomic variants the individual carries across the whole genome by effect sizes obtained from GWAS. PGSs thereby capture the polygenic signal of a given trait. ${ }^{28}$ Individuals with high PGSs carry more risk variants and are hypothesised to be more likely to develop a trait or disorder. Robust findings from GWAS are used to construct PGS for psychiatric disorders $^{29-31}$ and evaluate shared genetic risk between phenotypes. ${ }^{32-34}$

In patients followed up on average 5.3 years in quality registers covering specialized eating disorder care across Sweden, ${ }^{35}$ we defined two different outcomes: first, at the timepoint when the patients additionally joined the Anorexia Nervosa Genetics Initative (ANGI), we defined severity as the total score on the 16item Clinical Impairment Assessment (CIA) questionnaire. The CIA measures secondary psychosocial impairment due to eating disorder symptoms. Second, we created an index of SEED marked by a high CIA score plus a follow-up time $\geq 5$ years. Based on the literature, we selected three PGSs and explored their association with severity or a SEED: PGS for AN, SCZ, and BMI. First, we hypothesized that an individual's eating disorder severity and risk for a SEED would be influenced by a high AN PGS (i.e., higher genetic loading associated with greater severity). Second, based on the reported high genetic correlations between $A N$ and $S C Z,{ }^{27}$ the seven times increased risk of $S C Z$ and the familial liability of $S C Z$, in patients with eating disorders compared with the general population, ${ }^{23}$ we hypothesized that greater genetic liability to SCZ separately would be associated with greater severity and SEED. Furthermore, we chose the SCZ GWAS as it currently represents the best statistically powered GWAS of any psychiatric disorder genetically correlated with AN, shows high heritability, and may index genetic liability to general psychopathology. Third, given the observed negative genetic correlation between $\mathrm{AN}$ and $\mathrm{BMI}^{27}$ and the association between low phenotypic BMI and poor outcome, ${ }^{36-38}$ we hypothesized that a BMI PGS would separately be associated with severity and the development of a SEED. Furthermore, we chose the BMI PGS because it is currently the largest GWAS available of anthropometric traits genetically associated with AN, and, therefore, offers the greatest statistical power. Results could inform whether PGSs might serve as a useful adjunct tool in predicting severity or a SEED.

\section{Methods}

Sample. Our study includes Swedish AN cases from ANGI ${ }^{39}$ whose genetic analytic methods have been described. ${ }^{27}$ Participants were recruited for genotyping into ANGI via Swedish treatment centres and two national registers: (1) Riksät, the national quality register established in 1999, including patients treated for AN, BN, or eating disorders not otherwise specified (EDNOS) ${ }^{40}$ and (2) Stepwise, the internet-based clinical quality assurance database for specialized eating disorder care, established in 2005. Stepwise includes all Riksät and additional variables and was used by an expanding number of treatment centres until 2015. Patients are registered into Riksät/Stepwise if 1) they are medically/self-referred to a Swedish 
treatment centre, 2) treatment is intended, and 3) an eating disorder diagnosis has been established (Table 1). Patients were first entered into either register 1999-2016 and recontacted for participation in ANGI 2013-2016. Therefore, for some participants, the follow-up time between entering the treatment register and being recruited into ANGI was less than a month (Table 2). We calculated follow-up time as difference in years between year at first registration and the year when participants were re-contacted for recruitment into ANGI, covering a follow-up period of 0-16 years. Our analyses comprised a total of 2,843 individuals aged $10-66$ years with 45 males (1.6\% of the total sample; Table 2 ). 
Table 2

Descriptive statistics of quantitative demographic and clinical characteristics of target sample $(N=$ 2,843) of specialised eating disorder care in Sweden

\begin{tabular}{|c|c|c|c|c|c|c|c|}
\hline & & Mean & SD & Median & Min & Max & $\begin{array}{l}\text { Total } \\
\text { missing }\end{array}$ \\
\hline \multirow{8}{*}{$\begin{array}{l}\text { Self-reported } \\
\text { age at first ED } \\
\text { symptom }\end{array}$} & AN restricting only & 15.93 & 4.38 & 15 & 6 & 58 & \\
\hline & $\begin{array}{l}\text { AN binge- } \\
\text { eating/purging only }\end{array}$ & 15.64 & 4.13 & 15 & 6 & 44 & \\
\hline & $\begin{array}{l}\text { Broad AN only (without } \\
\text { amenorrhea and } \\
\text { weight criterion) }\end{array}$ & 15.31 & 3.78 & 15 & 6 & 45 & \\
\hline & AN + bulimia nervosa & 15.55 & 3.77 & 15 & 5 & 33 & \\
\hline & $\begin{array}{l}\text { AN + binge-eating } \\
\text { disorder }\end{array}$ & 17.31 & 6.74 & 16 & 10 & 55 & \\
\hline & AN + EDNOS & 15.68 & 4.30 & 15 & 6 & 36 & \\
\hline & $\begin{array}{l}\text { AN + purging disorder } \\
\text { or EDNOS } 5^{\star}\end{array}$ & 15.60 & 3.93 & 15 & 6 & 36 & \\
\hline & & & & & & & 155 \\
\hline \multirow{8}{*}{$\begin{array}{l}\text { Age at first } \\
\text { registration }\end{array}$} & AN restricting only & 19.44 & 6.68 & 18 & 10 & 61 & \\
\hline & $\begin{array}{l}\text { AN binge- } \\
\text { eating/purging only }\end{array}$ & 21.79 & 7.00 & 20 & 13 & 60 & \\
\hline & $\begin{array}{l}\text { Broad AN only (without } \\
\text { amenorrhea and } \\
\text { weight criterion) }\end{array}$ & 20.50 & 7.35 & 18 & 11 & 66 & \\
\hline & AN + bulimia nervosa & 25.33 & 7.98 & 23 & 13 & 66 & \\
\hline & $\begin{array}{l}\text { AN + binge-eating } \\
\text { disorder }\end{array}$ & 26.27 & 9.44 & 24 & 16 & 61 & \\
\hline & AN + EDNOS & 22.20 & 8.53 & 20 & 12 & 60 & \\
\hline & $\begin{array}{l}\text { AN + purging disorder } \\
\text { or EDNOS } 5^{\star}\end{array}$ & 23.21 & 8.62 & 21 & 11 & 60 & \\
\hline & & & & & & & 0 \\
\hline Body mass & AN restricting only & 15.91 & 1.39 & 16.01 & 12.05 & 21.63 & \\
\hline
\end{tabular}

*EDNOS 5 = Eating disorder not otherwise specified category 5, includes those patients that repeatedly chew and spit food, without swallowing, large amounts of food.

**Follow-up time was calculated as difference in years between year at first registration and the year when participants were re-contacted for recruitment into ANGI 


\begin{tabular}{|c|c|c|c|c|c|c|c|}
\hline & & Mean & SD & Median & Min & Max & $\begin{array}{l}\text { Total } \\
\text { missing }\end{array}$ \\
\hline & $\begin{array}{l}\text { AN binge- } \\
\text { eating/purging only }\end{array}$ & 16.52 & 1.49 & 16.61 & 12.95 & 25.07 & \\
\hline & $\begin{array}{l}\text { Broad AN only (without } \\
\text { amenorrhea and } \\
\text { weight criterion) }\end{array}$ & 18.63 & 1.89 & 18.46 & 13.5 & 30.7 & \\
\hline & AN + bulimia nervosa & 21.67 & 3.78 & 20.70 & 13.47 & 44.1 & \\
\hline & $\begin{array}{l}\text { AN + binge-eating } \\
\text { disorder }\end{array}$ & 24.38 & 6.35 & 22.48 & 15.8 & 43.56 & \\
\hline & AN + EDNOS & 19.63 & 4.4 & 18.85 & 12.74 & 40.39 & \\
\hline & $\begin{array}{l}\text { AN + purging disorder } \\
\text { or EDNOS } 5^{\star}\end{array}$ & 19.89 & 2.38 & 19.56 & 14.16 & 34.48 & \\
\hline & & & & & & & 15 \\
\hline \multirow{8}{*}{$\begin{array}{l}\text { ClA global } \\
\text { score }\end{array}$} & AN restricting only & 17.13 & 12.94 & 14 & 0 & 48 & \\
\hline & $\begin{array}{l}\text { AN binge- } \\
\text { eating/purging only }\end{array}$ & 21.17 & 14.19 & 21 & 0 & 48 & \\
\hline & $\begin{array}{l}\text { Broad AN only (without } \\
\text { amenorrhea and } \\
\text { weight criterion) }\end{array}$ & 18.58 & 12.73 & 17 & 0 & 48 & \\
\hline & AN + bulimia nervosa & 19.84 & 12.83 & 19 & 0 & 48 & \\
\hline & $\begin{array}{l}\text { AN + binge-eating } \\
\text { disorder }\end{array}$ & 19.33 & 13.84 & 21 & 0 & 46 & \\
\hline & AN + EDNOS & 14.51 & 11.3 & 11 & 0 & 40 & \\
\hline & $\begin{array}{l}\text { AN + purging disorder } \\
\text { or EDNOS } 5^{\star}\end{array}$ & 19.88 & 13.17 & 20 & 0 & 48 & \\
\hline & & & & & & & 0 \\
\hline \multirow{3}{*}{$\begin{array}{l}\text { Follow-up } \\
\text { time }\end{array}$} & AN restricting only & 5.31 & 3.34 & 5 & 0 & 15 & \\
\hline & $\begin{array}{l}\text { AN binge- } \\
\text { eating/purging only }\end{array}$ & 5.38 & 3.64 & 5 & 0 & 14 & \\
\hline & $\begin{array}{l}\text { Broad AN only (without } \\
\text { amenorrhea and } \\
\text { weight criterion) }\end{array}$ & 4.59 & 3.25 & 4 & 0 & 16 & \\
\hline
\end{tabular}

*EDNOS 5 = Eating disorder not otherwise specified category 5, includes those patients that repeatedly chew and spit food, without swallowing, large amounts of food.

**Follow-up time was calculated as difference in years between year at first registration and the year when participants were re-contacted for recruitment into ANGI 


\begin{tabular}{|c|c|c|c|c|c|c|}
\hline & Mean & SD & Median & Min & Max & $\begin{array}{l}\text { Total } \\
\text { missing }\end{array}$ \\
\hline AN + bulimia nervosa & 5.19 & 3.69 & 4 & 0 & 16 & \\
\hline $\begin{array}{l}\text { AN + binge-eating } \\
\text { disorder }\end{array}$ & 5.58 & 3.55 & 5 & 0 & 14 & \\
\hline AN + EDNOS & 4.34 & 2.82 & 3 & 0 & 12 & \\
\hline $\begin{array}{l}\text { AN + purging disorder } \\
\text { or EDNOS } 5^{\star}\end{array}$ & 3.91 & 3.14 & 3 & 0 & 14 & \\
\hline & & & & & & 0 \\
\hline \multicolumn{7}{|c|}{$\begin{array}{l}\text { *EDNOS } 5 \text { = Eating disorder not otherwise specified category } 5 \text {, includes those patients that } \\
\text { repeatedly chew and spit food, without swallowing, large amounts of food. }\end{array}$} \\
\hline
\end{tabular}

Clinical diagnosis. To be classified as a case in ANGI, a lifetime DSM-IV AN diagnosis (amenorrhea was not required) was confirmed based on answers to the ED100K-v1 questionnaire ${ }^{39}$ or by a clinical AN diagnosis registered in the national registers. Importantly, all individuals in our study had a lifetime AN diagnosis, but may also have been diagnosed with an additional eating disorder. This means we could delineate a persistent AN group from a AN with mixed eating disorder presentation group, representing the diagnostic crossover common in eating disorders ${ }^{41}$ (Table 1). In Riksät, diagnoses are based on clinician interviews and clinical observation until 2013 when the DSM-IV-based Structured Eating Disorder Interview (SEDI) ${ }^{42}$ was also implemented. In Stepwise, the Structured Clinical Interview for DSM-IV Axis I disorders (SCID-I), eating disorder diagnostic research version (module $H)^{43}$ for adults $\geq 18$ years was administered from 2005 until August 2008 alongside the MINIkid version 2.1 for DSM-IV (i.e., Modules S and T $)^{44}$ for individuals $<18$ years. The DSM-IV-based SEDI ${ }^{42}$ has been used since August 2008. 
Table 1

Lifetime eating disorder diagnoses registered in Swedish quality registers Riksät/Stepwise among individuals with a lifetime diagnosis of anorexia nervosa (AN) included in our target sample $(n=2,834)$

\section{Eating disorder diagnoses registered in Stepwise/Riksät}

\begin{tabular}{|lll|}
\hline & $n$ (cases) & $\%$ of total \\
\hline AN restricting only & 976 & 34.3 \\
\hline AN binge-eating/purging only & 260 & 9.2 \\
\hline Broad AN only (without amenorrhea and weight criterion) & 603 & 21.2 \\
\hline AN + bulimia nervosa & 522 & 18.4 \\
\hline AN + binge-eating disorder & 45 & 1.6 \\
\hline AN + EDNOS & 95 & 3.3 \\
\hline AN + purging disorder or EDNOS 5* & 342 & 12.0 \\
\hline Total & 2843 & \\
\hline $\begin{array}{l}\text { Note. AN = anorexia nervosa, BN = bulimia nervosa, BED = binge-eating disorder, EDNOS = eating } \\
\text { disorder not otherwise specified, } P D=\text { purging disorder }\end{array}$ & \\
\hline
\end{tabular}

Treatment centres. We grouped the 41 treatment centres into five categories reflecting their geographic locations across Sweden. We excluded Stockholm from "Svealand" to create a more equal distribution of participants across categories, reflecting differences between rural and urban regions. Most patients were first registered in the region of Stockholm (32.3\%), followed by Västra Götaland (22.3\%), Östergötland (22.0\%), and Svealand (16.0\%), while the fewest patients were registered in Norrland (7.4\%). About $29 \%$ of patients at treatment centres in Stockholm received an AN restricting diagnosis.

Ethics. All participants provided written informed consent for participation in ANGI. The Swedish component of ANGI was approved by the regional Ethical Review Board in Stockholm (dnr: 2013/112$31 / 2)$ and the amendments (2014/1563 and 2016/1852-32).

Eating disorder severity as measured by the Clinical Impairment Assessment. The 16-item questionnaire measures the severity of secondary psychosocial impairment due to eating disorder features during the previous 28 days ${ }^{35}$ covering three domains: emotional, social, and cognitive functioning. Each item is rated on a 4-point Likert scale with $0=$ 'not at all', $1=$ 'a little', $2=$ 'quite a bit', and $3=$ 'a lot'. Summing all items yields a total severity score ranging from 0 to 48 , with higher scores indicating greater impairment (Supplementary Figures S1A-C). More details on the questionnaire are in the Supplementary Methods. Correlations between items were assessed using polychoric correlations and polyserial correlations between the sum scores (i.e., global and domain-specific scores) and the 16 items. ${ }^{45}$ Pearson's correlations were used to estimate the association between the sum scores (Supplementary Figure S2). 
SEED definition. With the assumption that a CIA total score $\geq 18$ is associated with case status, ${ }^{46}$ we defined our binary dependent SEED variable as CIA score $\geq 18$ and a follow-up time $\geq 5$ years (i.e., years between initial registration and ANGI recruitment) independent of eating disorder diagnosis or diagnostic crossover. We compared patients with a SEED with those having a CIA score $<18$ and follow-up time $\geq 5$ years in logistic regressions. As treatment resistance is part of the proposed definition of a SEED ${ }^{7}$ it is important to note that all patients registered in Risksät/Stepwise have undergone at least one treatment attempt, qualifying them for a SEED.

PGS calculation: clumping and thresholding. The PGSs for AN (after excluding Swedish participants; $n=$ $4118)^{39}$ and $S C Z^{31}$ were based on genome-wide association data from the Psychiatric Genomics Consortium (PGC), and a PGS for BMI based on data from the Genetic Investigation of ANthropometric Traits (GIANT) consortium and the UK Biobank. ${ }^{47}$ SNPs that were present exclusively in either the GWAS summary statistics or the ANGI dataset as well as ambiguous SNPs were removed before PGS calculation using PRSice, version 2.2.3. ${ }^{48}$ Linkage disequilibrium (LD) among SNPs was accounted for by clumping. The genetically independent SNP with the smallest $p$ value in each 250 kilobase window of all those in LD was retained as the index SNP $\left(r^{2}>0.1\right)$. We calculated PGSs at different $p$ value thresholds ( $p$ values: $\left.5 \times 10^{-8}, 1 \times 10^{-5}, 0.001,0.01,0.05,0.1,0.2,0.3,0.4,0.5,1\right)$ for sensitivity analyses. The main results are based on the PGSs at a $p$ value threshold of 0.001 as this PGS explained most of the variance in the model including the AN PGS $\left(\mathrm{R}^{2}=0.175 \%\right)$. We also chose to report this threshold in the models including the BMI or SCZ PGSs to facilitate comparison across models. As sensitivity analysis, we present results for all other $p$ value thresholds. We included standardised PGS as continuous variables, reporting results as one unit change per one standard deviation higher PGS.

Polygenic risk score continuous shrinkage (PRS-CS). We observed inconsistent results for the AN PGS across the different $p$ value thresholds at which they were generated. Therefore, we used PRS-CS as a sensitivity analysis to further investigate the robustness of our results. PRS-CS does not rely on $p$ value thresholding and is a Bayesian approach to calculate PGS. It applies two shrinkage parameters to the original effect sizes of the discovery GWAS: a global scaling parameter shared across all effect sizes and a local, marker-specific parameter, resulting in a global-local scale mixtures of normals. In an extreme case, where the local shrinkage would be one, the model would be a Ridge regression and all effect sizes were shrunk by the global parameter only. However, the local shrinkage parameter allows heterogeneity in the scales of the effect sizes and the global parameter controls the sparsity of the model. In other words, small effects are shrunk towards zero, but large effect sizes are less affected. In summary, the PRS-CS method calculates a reweighted PGS assuming a different degree of involvement of different SNPS across the genome. No selection of a $p$ value threshold is needed. The re-weighted PGS was used as an explanatory variable in our regression models. Correlations between the thresholding-based and PRS-CSbased PGS are presented in Supplementary Figures S3A-C. 
Table 3

Regression models fitted. Model 3 is the main model in the logistic regression analysis and model 4 is the main model in the linear regression. The other models are sensitivity analyses.

\begin{tabular}{|c|c|c|c|}
\hline $\begin{array}{l}\text { Linear } \\
\text { regression }\end{array}$ & $\begin{array}{l}\text { Logistic } \\
\text { regression }\end{array}$ & Variables & Justification \\
\hline Model 1 & Model 1 & $\begin{array}{l}\text { CIA or SEED PGS + PCs } \\
1-10+\text { treatment region }\end{array}$ & Population stratification and nesting \\
\hline Model 2 & Model 2 & Model $1+$ Age & $\begin{array}{l}\text { Older age at treatment initiation is } \\
\text { associated with poor outcome. }{ }^{49}\end{array}$ \\
\hline Model 3 & $\begin{array}{l}\text { Model } 3 \\
\text { (main } \\
\text { model) }\end{array}$ & $\begin{array}{l}\text { Model } 2+\mathrm{Sex}+\mathrm{AN} \\
\text { presentation* }\end{array}$ & $\begin{array}{l}\text { Sex differences in presentation }{ }^{50} \text { and } \\
\text { prevalence }{ }^{51} \text { of eating disorders }\end{array}$ \\
\hline $\begin{array}{l}\text { Model } 4 \\
\text { (main } \\
\text { model) }\end{array}$ & - & $\begin{array}{l}\text { Model } 3+\text { Follow-up } \\
\text { time }\end{array}$ & $\begin{array}{l}\text { To evaluate disorder severity independent of } \\
\text { follow-up time (years between first } \\
\text { registration and ANGI recruitment). }\end{array}$ \\
\hline Model 5 & Model 4 & $\begin{array}{l}\text { Model } 3+\text { Follow-up } \\
\text { time** }+ \text { BMl }\end{array}$ & $\begin{array}{l}\text { Low body weight as predictor of poor } \\
\text { outcome. }{ }^{44,15,22}\end{array}$ \\
\hline Model 6 & Model 5 & $\begin{array}{l}\text { Model } 3+\text { Follow-up } \\
\text { time }{ }^{\star \star}+\text { Self-reported } \\
\text { age at first ED symptom }\end{array}$ & $\begin{array}{l}\text { Early age at onset is indicative of a better } \\
\text { outcome } e^{22} \text { and hormonal changes are } \\
\text { associated with disorder onset. } 52\end{array}$ \\
\hline \multicolumn{4}{|c|}{$\begin{array}{l}\text { Note. } A N G I=\text { Anorexia Nervosa Genetics Initiative, } C I A=\text { Clinical Impairment Assessment, }{ }^{35} E D= \\
\text { eating disorder, } B M I=\text { body mass index, } P C=\text { principal component, } P G S=\text { polygenic score, } S E E D= \\
\text { severe and enduring eating disorder, }\end{array}$} \\
\hline \multicolumn{4}{|c|}{$\begin{array}{l}\text { *Only adjusted for eating disorder diagnosis when including all participants, irrespective of clinically } \\
\text { ascertained eating disorder diagnosis additional to AN. }\end{array}$} \\
\hline
\end{tabular}

Statistical analysis. We associated the three PGS for AN, SCZ, and BMI with disorder severity measured by the CIA total score as a continuous dependent variable (i.e., linear regression) and with SEED (i.e., logistic regression), resulting in six regression models. To avoid collinearity among independent variables, we excluded highly correlated variables (i.e., $r>0.80$; Supplementary Figure S4). In our main model (Table 3), we fitted the PGS as the independent variable and adjusted for potential population stratification by including the first 10 ancestry-informative principal components (PCs), and controlled for nesting within our sample by including treatment region. ${ }^{53}$ Using PLINK 2.0, we calculated PCs based on pruned genotype data. ${ }^{54}$ Additionally, we included age, sex, and follow-up time. For all model details including justification, see Table 3. Mendelian randomisation studies imply a bidirectional causal relationship between $\mathrm{AN}$ and low BMl; hence, adjusting for BMI in models including patients with AN may introduce a collider. ${ }^{27}$ Therefore, we investigated BMI in an additional model (logistic model 4 and linear model 5). 
We investigated three different polygenic scores and two outcomes and therefore adjusted our $a$ threshold using the Bonferroni method $\alpha=\frac{0.05}{6}=0.008$ to account for the multiple tests performed.

Exclusion. From analysis including $\mathrm{BMI}$ as a covariate, 15 patients were excluded due to missing data, and 155 patients were excluded because of missing retrospectively self-reported age at first eating disorder symptom. From the logistic models, we excluded follow-up time as a covariate since follow-up time is part of the SEED outcome definition (i.e., longer than 5 years). This means that the logisitic regression only includes patients with a follow-up $\geq 5$ years. Therefore, the total sample size for the logistic regression analysis was $n=1,334$.

Sensitivity analyses. Approximately $35 \%$ of the individuals in our target sample (Table 1 ) were not diagnosed with AN when they were first registered into Riksät/Stepwise. Because this mixed presentation of an eating disorder in one individual may influence the association between PGS and outcome, we used sensitivity analyses to evaluate the effect of diagnostic heterogeneity in the eating disorder presentation. We created four different samples: first, the whole sample including individuals with AN and a mixed presentation of other eating disorder diagnoses, second, individuals with AN, including a clinical AN restricting, binge-eating/purging subtype, or AN without amenorrhea and weight criterion, third, only individuals with a clinical AN restricting diagnosis, as this may represent a unique phenotype, fourth, only individuals with AN (both subtypes) with a low BMI ( $\left.\leq 17.5 \mathrm{~kg} / \mathrm{m}^{2}\right)$. The subsamples including all AN subtypes or only the AN restricting subtype did not require adjustment for the eating disorder diagnosis. In this case, we fitted five logistic regression models.

\section{Results}

Participants with AN restricting subtype had the lowest average BMI $\left(15.9 \mathrm{~kg} / \mathrm{m}^{2}\right)$, were the youngest (age $=19.4$ years) at first registration, and had the lowest CIA total score (mean CIA $=17$; i.e., least severe). Self-reported age at first eating disorder symptom was similar across all groups except for individuals with lifetime co-occurring BED, who reported the oldest age at experiencing their first eating disorder symptom (age $=17.3$ years). Follow-up time was on average the same across all groups (follow-up time $\approx 5$ years) except for participants with AN plus purging disorder (follow-up time $=3.91$ years; Table 2 ). Supplementary Figures $1 \mathrm{~A}-\mathrm{C}$ summarise responses to each $\mathrm{CIA}$ item in all three samples.

\section{Association of PGSs with eating disorder severity as measured by the CIA}

AN PGS and eating disorder severity. After multiple testing correction $(\alpha=0.008)$, the AN PGS was not associated with disorder severity (Supplementary Table S1) measured as the CIA total score in either the full sample with clinically ascertained AN cases with a mixed presentation $(n=2,843$, Figure $1 \mathrm{~A})$, or the AN cases subsample (i.e., AN restricting, AN binge-eating/purging, Broad AN; $n=1,839$, Figure 1B), or the subsample of individuals with the AN restricting subtype ( $n=976$, Figure $1 C)$, or the subsample of individuals with low weight AN. Adjustment of our models did not change the association between the AN 
PGS and the CIA total score. Moreover, after shrinking the effect sizes and re-weighting the PGS with PRS$\mathrm{CS}$, the association remained non-significant (Supplementary Table S2)

SCZ PGS and eating disorder severity. The same result was observed for the SCZ PGS: no association remained significant at the $\alpha=0.008$ (Figures 2A-C and Supplementary Table S3). We did not observe any significant association by subsample, model adjustment, or PRS calculation method.

BMI PGS and eating disorder severity. The BMI PGS showed a positive association with eating disorder severity as measured by the CIA (Figure 3 and Supplementary Table S4). In the full sample including clinical AN cases with a mixed presentation, one standard deviation greater BMI PGS was associated with a 0.83 ( $95 \% \mathrm{Cl}: 0.36,1.30 ; p=5.5 \times 10^{-4}$ ) greater CIA total score (Figure 3A). Limiting the sample to AN only cases strengthend the association: one standard deviation greater BMI PGS was associated with 1.30 (95\% Cl: $\left.0.72,1.88 ; p=1.2 \times 10^{-5}\right)$ greater CIA total score (Figure $3 \mathrm{~B}$ ). In the AN restricting subtype subsample or the low weight AN subsample, the association did not remain significant at our a threshold (Figure $3 C$ ). The results in the full sample and the AN only sample were consistent across all $p$ value thresholds at which the PGS was generated when using clumping and thresholding. Additionally, the results remained largely the same with varying adjustment of our models. However, after shrinking the effect sizes and re-weighting the PGS with PRS-CS, the association did not remain significant

(Supplementary Table S2)

\section{Association Of Pgs With Risk Of Seed}

We tested if PGSs for any of the three selected traits were associated with SEED, defined as those individuals with a CIA score $\geq 18$ and a follow-up time $\geq 5$ years.

AN PGS and SEED. In the full sample with a mixed AN presentation, the AN PGS was not significantly associated with a SEED ( $n=1,334$ Figure $4 \mathrm{~A})$. When we restricted the analysis to individuals with AN only (i.e., AN restricting, AN binge-eating/purging, Broad AN; $n=901$ ), a one standard deviation greater AN PGS was associated with $24 \%$ higher odds of being classified as a SEED ( $95 \% \mathrm{Cl}: 1.08,1.43 ; p=0.002$; Figure $4 \mathrm{D}$ and Supplementary Table S5). The association did not remain significant in the AN restricting subsample ( $n=509$; Figure $4 \mathrm{G}$ ) or the low weight subsample. We detected the association only at a PGS generation $p$ value threshold of 0.001 ; however, the association was independent of adjustment by other variables. The association was not detected when calculating the PGS with PRS-CS.

SCZ PGS and SEED. We found no statistically significant associations between SCZ PGSs and SEED in either the full or our subsamples of diagnostic subgroups (Figure 4B, 4E, 4H and Supplementary Table S5). Model adjustement or a different PGS calculation method did not change the result.

BMI PGS and SEED. We found no statistically significant associations between BMI PGSs and SEED in either the full or our subsamples of diagnostic subgroups at our main PGS generation $p$ value threshold of 0.001 . However, the BMI PGS was significantly associated with SEED only in the full sample with a 
mixed AN presentation with OR ranging from 1.19 to 1.23; Figure 4C and Supplementary Table S6). We observed this association only when including self-reported age at first eating disorder symptom (model 5) as an additional explanatory variable and when including more SNPs in the PGS (i.e., generation $p$ value thresholds from 0.05 until 1). Overall, these results were inconsistent.

\section{Discussion}

Eating disorders are often chronic ${ }^{4}$ and no biomarkers exist that identify individuals at risk for an eating disorder, let alone for a protracted illness course. Our results suggest that a PGS indicating genetic liability to BMI may be a useful adjunct tool in predicting who is at risk for developing a severe an enduring form of the illness. Additionally, if the sample size for the AN GWAS increases, and the AN PGS may become more statistically powerful, the AN PGS should be re-assessed for outcome prediction.

Several recent reviews have highlighted the potential clinical application of PGS in psychiatry, as well as cautions about their overinterpretation. ${ }^{55,56}$ Research on other psychiatric disorders such as depression has shown that the cumulative influence of multiple genetic variants is associated with chronic depressive symptoms. ${ }^{57}$ Similarly, a high SCZ PGS is associated with poor treatment response, suggesting that polygenic burden may impact treatment resistance. ${ }^{58}$

Our results represent the first indication that an AN PGS may have clinical utility in the future. PGSs have become increasingly robust as the sample sizes of their source GWASs increase. The AN GWAS is still fairly immature, and in addition, our polygenic score had less power as we had to exclude the Swedish participants ( $n=4,118$ of 16,992 cases) from the discovery sample. Nevertheless, it had predictive capacity in our sample, especially in those individuals who were firmly anchored in an AN diagnosis only. The effect appears to be diluted in those individuals who display a more mixed eating disorders diagnostic picture across their illness journey. This may limit potential clinical utility in this subgroup of patients. The observed difference between those with AN only and those with a more mixed diagnostic picture also provides insight into how genetic factors may influence clinical presentation and course within the eating disorder diagnostic groups. These results are consistent with prior observations suggesting that three primary eating disorders (AN, BN, and BED) differ on a genomic level. ${ }^{59}$ AN PGS at initial presentation for treatment may hold promise in predicting who is likely to maintain an AN presentation versus experience diagnostic crossover, informing the tailoring of treatment accordingly. This should be investigated in larger independent samples as our results were inconsitent.

In our study, genetic liability to SCZ was not associated with disorder severity or long-term outcome in patients with AN. However, in the subsample of AN restricting patients only, the SCZ PGS showed a nominally significant (i.e., $\mathrm{p}<0.05$ ) negative association with the CIA score. This association did not remain significant after multiple testing correction, and was not detected at the main analysis $p$ value threshold to generate the PGS of 0.001 . The associations overall were inconsistent. Potentially, a larger target sample size providing greater statistical power may clarify these findings. The reason for a potential association between a higher SCZ polygenic load with a lower CIA score (i.e., less self-reported 
severity) is unclear, but genetic liability to SCZ could possibly be associated with poor illness insight, sometimes termed unintentional denial of illness or anosognosia, which is common in AN and often evidenced by unexpectedly low self-report of symptoms and distress relative to objective illness status. ${ }^{60,61}$ Future research could investigate further whether AN patients with high SCZ PGS represent a distinct phenotype with different treatment needs and response.

In our study, patients with AN carrying a greater BMI PGS experienced greater severity compared to eating disorder patients with low BMI PGSs. This finding stands in contrast to negative genetic correlation between $\mathrm{BMI}$ and $\mathrm{AN}$ as a diagnosis based on GWAS data ${ }^{27,59,62}$. However, the positive association between the BMI PGS and severity is consistent with findings that associated genetic liability for high $\mathrm{BMI}$ with engaging in more weight loss behaviours ${ }^{63}$ and higher levels of disordered eating ${ }^{64}$ in the general population. These studies ${ }^{63,64}$ and our finding support a shared genetic aetiology between genetic propensity for high BMI and eating disorder severity or specific symptoms. As fear of weight gain, a central symptom among individuals with some eating disorders, combined with genetic liability for high BMI might induce psychological distress, reflected as higher scores on the CIA in our study, future studies should investigate more refined measures of severity on the symptom level. Additionaly, our finding needs to be replicated in an independent sample.

\section{Limitations}

Our findings must be interpreted in the light of several limitations. First, eating disorder diagnoses are entered at first registration to the national quality registers, meaning that some diagnostic crossover could have occurred prior to or after registration that was not captured in our data. Mitigating this limitation was the addition of the questionnaire-based lifetime data collected in the ED100K as part of the ANGI study that captures lifetime diagnostic migration. Second, we only included individuals with eating disorders who sought treatment. Although this increases clinical applicability, this might also have resulted in a more severe sample with less variance. Balancing that limitation, we included data from the majority of specialized eating disorder units across Sweden, capturing almost all Swedish individuals diagnosed with an eating disorder receiving all levels of care. Third, our measure of disorder severity is limited as we only used one continuous score at one time point. Future studies should include longitudinal assessments of severity and potentially pool different outcome variables to create a composite measure of severity. Fourth, participants were primarily of European (and Swedish) ancestry, which limits the generalisability to non-European populations. Fifth, the low number of males included in this study hinders our ability to address sex differences. Sixth, as the AN PGS was derived from a comparatively small GWAS excluding the Swedish participants, our statistical power to detect associations was limited. This is refelected in the inconsistent effect estimates across different $p$ value thresholds at which the AN PGS was created (Figure 1A-C) and additionally the inconsistent results when using the PRS-CS method to calculate PGSs. This phenomenon, however, had also been observed in studies of SCZ. ${ }^{65}$ As AN most likely is polygenic and may have diverse underlying causes, one may hypothesise that more strongly associated genetic variants (i.e., lower generation $p$ value thresholds) act 
via different biological pathways than less associated ones. ${ }^{66}$ However, findings are mixed. ${ }^{65,66}$ It is anticipated that increasing the sample size of eating disorders GWASs will lead to the identification of more AN-associated genetic variants, as seen in other psychiatric disorders such as $\mathrm{SCZ},{ }^{67}$ boosting the statistical power, robustness, and clinical utility of the AN PGS. ${ }^{27}$ Analyses like ours should be repeated when PGS with greater statistical power are available. Furthermore, different genetic variants may be implicated in the development of than in the maintenance of AN. Therefore, future GWASs should not only focus on a binary disorder phenotype, but they should also consider the course of the disorder along the lifespan.

\section{Conclusion}

No study of long-term outcome of eating disorders has included the role of genomic risk burden. Our study is the first to show an association between an AN and BMI PGS with severity or eating disorder outcome. We provide the first tentative evidence of potential clinical utility of PGSs ${ }^{68}$ in the field of eating disorders. Our findings suggest that PGSs in combination with environmental variables may contribute to risk prediction models in AN, if GWAS sample sizes continue to increase. Ongoing concerns with slow progress in improving treatment outcomes, especially for $\mathrm{AN}^{69,70}$ call for advances in prevention, detection, and treatment. These results encourage the expansion of genetic studies of eating disorders to accelerate discovery and impact.

\section{Declarations}

\section{Declaration of interest}

CM Bulik reports: Shire (grant recipient, Scientific Advisory Board member); Idorsia (consultant); Lundbeckfonden (grant recipient); Pearson (author, royalty recipient). All other authors have indicated they have no conflicts of interest to disclose.

\section{Acknowledgements}

The Anorexia Nervosa Genetics Initiative is an initiative of the Klarman Family Foundation. We acknowledge the assistance of the Stockholm Centre for Eating Disorders (SCÄ), and thank Lifegene and the Swedish National Quality Register for Eating Disorders (Riksät) for assistance in data collection as well as long-term follow-up CIA data. We would also like to thank the research nurses and data collectors at the Department of Medical Epidemiology and Biostatistics who worked on ANGI.

\section{Code availability}

Code to generate results is available upon request from the authors and made available on GitHub with publication.

\section{References}


1. Yilmaz, Z., Hardaway, J. A. \& Bulik, C. M. Genetics and Epigenetics of Eating Disorders. Adv. Genomics Genet. 5, 131-150 (2015).

2. Arcelus, J., Mitchell, A. J., Wales, J. \& Nielsen, S. Mortality rates in patients with anorexia nervosa and other eating disorders. A meta-analysis of 36 studies. Arch. Gen. Psychiatry 68, 724-731 (2011).

3. Fichter, M. M., Quadflieg, N., Crosby, R. D. \& Koch, S. Long-term outcome of anorexia nervosa: Results from a large clinical longitudinal study. Int. J. Eat. Disord. 50, 1018-1030 (2017).

4. Steinhausen, H.-C. Outcome of eating disorders. Child Adolesc. Psychiatr. Clin. N. Am. 18, 225-242 (2009).

5. Noordenbos, G., Oldenhave, A., Muschter, J. \& Terpstra, N. Characteristics and Treatment of Patients with Chronic Eating Disorders. Eat. Disord. 10, 15-29 (2002).

6. Long, C. G., Fitzgerald, K.-A. \& Hollin, C. R. Treatment of chronic anorexia nervosa: a 4-year followup of adult patients treated in an acute inpatient setting. Clin. Psychol. Psychother. 19, 1-13 (2012).

7. Robinson, P. H. Severe and Enduring Eating Disorder. (John Wiley \& Sons, Ltd, 2009).

8. Andries, A., Frystyk, J., Flyvbjerg, A. \& Støving, R. K. Dronabinol in severe, enduring anorexia nervosa: a randomized controlled trial. Int. J. Eat. Disord. 47, 18-23 (2014).

9. Fox, J. R. E. \& Diab, P. An exploration of the perceptions and experiences of living with chronic anorexia nervosa while an inpatient on an Eating Disorders Unit: An Interpretative Phenomenological Analysis (IPA) study. J. Health Psychol. 20, 27-36 (2013).

10. Dawson, L., Rhodes, P. \& Touyz, S. "Doing the Impossible": The Process of Recovery From Chronic Anorexia Nervosa. Qual. Health Res. 24, 494-505 (2014).

11. Touyz, S. et al. Treating severe and enduring anorexia nervosa: a randomized controlled trial. Psychol. Med. 43, 2501-2511 (2013).

12. Arkell, J. \& Robinson, P. A pilot case series using qualitative and quantitative methods: biological, psychological and social outcome in severe and enduring eating disorder (anorexia nervosa). Int. J. Eat. Disord. 41, 650-656 (2008).

13. Keski-Rahkonen, A. et al. Factors associated with recovery from anorexia nervosa: A populationbased study. Int. J. Eat. Disord. 47, 117-123 (2014).

14. Zipfel, S., Lowe, B., Reas, D. L., Deter, H. C. \& Herzog, W. Long-term prognosis in anorexia nervosa: lessons from a 21-year follow-up study. Lancet (London, England) vol. 355 721-722 (2000).

15. Löwe, B. et al. Long-term outcome of anorexia nervosa in a prospective 21-year follow-up study. Psychol. Med. 31, 881-890 (2001). 
16. Lock, J. et al. Do end of treatment assessments predict outcome at follow-up in eating disorders? Int. J. Eat. Disord. 46, 771-778 (2013).

17. Keel, P. K., Mitchell, J. E., Miller, K. B., Davis, T. L. \& Crow, S. J. Long-term Outcome of Bulimia Nervosa. Arch. Gen. Psychiatry 56, 63-69 (1999).

18. Keel, P. K. \& Brown, T. A. Update on course and outcome in eating disorders. Int. J. Eat. Disord. 43, 195-204 (2010).

19. Bulik, C. M., Sullivan, P. F., Joyce, P. R., Carter, F. A. \& Mclntosh, V. V. Predictors of 1-year treatment outcome in bulimia nervosa. Compr. Psychiatry 39, 206-214 (1998).

20. Robinson, P. Severe and enduring eating disorders: recognition and management. Adv. Psychiatr. Treat. 20, 392-401 (2014).

21. Berkman, N. D., Lohr, K. N. \& Bulik, C. M. Outcomes of eating disorders: a systematic review of the literature. Int. J. Eat. Disord. 40, 293-309 (2007).

22. Steinhausen, H.-C. The outcome of anorexia nervosa in the 20th century. Am. J. Psychiatry 159, 1284-1293 (2002).

23. Zhang, R. et al. Familial co-aggregation of schizophrenia and eating disorders in Sweden and Denmark. Mol. Psychiatry (2020) doi:10.1038/s41380-020-0749-x.

24. Foulon, C. [Schizophrenia and eating disorders]. Encephale. 29, 463-466 (2003).

25. Khalil, R. B., Hachem, D. \& Richa, S. Eating disorders and schizophrenia in male patients: A review. Eat. Weight Disord. - Stud. Anorexia, Bulim. Obes. 16, e150-e156 (2011).

26. Götestam, K. G., Eriksen, L. \& Hagen, H. An epidemiological study of eating disorders in Norwegian psychiatric institutions. Int. J. Eat. Disord. 18, 263-268 (1995).

27. Watson, H. J. et al. Genome-wide association study identifies eight risk loci and implicates metabopsychiatric origins for anorexia nervosa. Nat. Genet. 51, 1207-1214 (2019).

28. Maier, R. M., Visscher, P. M., Robinson, M. R. \& Wray, N. R. Embracing polygenicity: A review of methods and tools for psychiatric genetics research. Psychol. Med. 48, 1055-1067 (2018).

29. Howard, D. M. et al. Genome-wide meta-analysis of depression identifies 102 independent variants and highlights the importance of the prefrontal brain regions. Nat. Neurosci. 22, 343-352 (2019).

30. Stahl, E. A. et al. Genome-wide association study identifies 30 loci associated with bipolar disorder. Nat. Genet. 51, 793-803 (2019). 
31. Ripke, S. et al. Biological insights from 108 schizophrenia-associated genetic loci. Nature 511, 421-427 (2014).

32. Bulik-Sullivan, B. et al. An atlas of genetic correlations across human diseases and traits. Nat. Genet. 47, 1236-1241 (2015).

33. Smoller, J. W. et al. Psychiatric genetics and the structure of psychopathology. Mol. Psychiatry 24, 409-420 (2019).

34. Bauer, A. E. et al. Genetic risk scores for major psychiatric disorders and the risk of postpartum psychiatric disorders. Transl. Psychiatry 9, 288 (2019).

35. Bohn, K. et al. The measurement of impairment due to eating disorder psychopathology. Behav. Res. Ther. 46, 1105-1110 (2008).

36. Mayer, L. E. S. et al. Does percent body fat predict outcome in anorexia nervosa? Am. J. Psychiatry 164, 970-972 (2007).

37. Bodell, L. P. \& Mayer, L. E. S. Percent body fat is a risk factor for relapse in anorexia nervosa: a replication study. Int. J. Eat. Disord. 44, 118-123 (2011).

38. El Ghoch, M., Calugi, S., Chignola, E., Bazzani, P. V \& Dalle Grave, R. Body mass index, body fat and risk factor of relapse in anorexia nervosa. Eur. J. Clin. Nutr. 70, 194-198 (2016).

39. Thornton, L. M. et al. The Anorexia Nervosa Genetics Initiative (ANGI): Overview and methods. Contemp. Clin. Trials 74, 61-69 (2018).

40. Birgegard, A., Bjorck, C. \& Clinton, D. Quality assurance of specialised treatment of eating disorders using large-scale Internet-based collection systems: methods, results and lessons learned from designing the Stepwise database. Eur. Eat. Disord. Rev. 18, 251-259 (2010).

41. Schaumberg, K. et al. Patterns of diagnostic transition in eating disorders: a longitudinal population study in Sweden. Psychol. Med. 49, 819-827 (2019).

42. Birgegård, A. \& De Man Lapidoth, J. Validation of the structured eating disorder interview (SEDI) against the eating disorder examination (EDE). Karolinska Institutet (2010).

43. First, M. B., Gibbon, M., Spitzer, R. L., \& Williams, J. B. W. Structured clinical interview for DSM-IV Axis I disorders (J. Herlofson Trans.). Danderyd: Pilgrim Press. ((J. Herlofson Trans.). Danderyd: Pilgrim Press., 1998).

44. Kearney, C. A., Freeman, A. \& Bacon, V. 11 - Structured and semistructured interviews for children. in (eds. Goldstein, G., Allen, D. N. \& DeLuca, J. B. T.-H. of P. A. (Fourth E.) 337-353 (Academic Press, 2019). doi:https://doi.org/10.1016/B978-0-12-802203-0.00011-0. 
45. Holgado-Tello, F. P., Chacón-Moscoso, S., Barbero-García, I. \& Vila-Abad, E. Polychoric versus Pearson correlations in exploratory and confirmatory factor analysis of ordinal variables. Qual. Quant. 44, 153 (2008).

46. Ekeroth, K. \& Birgegård, A. Author 's personal copy Evaluating reliable and clinically signi fi cant change in eating disorders: Comparisons to changes in DSM-IV diagnoses.

47. Yengo, L. et al. Meta-analysis of genome-wide association studies for height and body mass index in 700000 individuals of European ancestry. Hum. Mol. Genet. 27, 3641-3649 (2018).

48. Choi, S. W. \& O'Reilly, P. F. PRSice-2: Polygenic Risk Score software for biobank-scale data. Gigascience 8, (2019).

49. Ackard, D. M., Richter, S., Egan, A. \& Cronemeyer, C. Poor outcome and death among youth, young adults, and midlife adults with eating disorders: an investigation of risk factors by age at assessment. Int. J. Eat. Disord. 47, 825-835 (2014).

50. Kinasz, K., Accurso, E. C., Kass, A. E. \& Le Grange, D. Does Sex Matter in the Clinical Presentation of Eating Disorders in Youth? J. Adolesc. Health 58, 410-416 (2016).

51. Striegel-Moore, R. H. et al. Gender difference in the prevalence of eating disorder symptoms. Int. J. Eat. Disord. 42, 471-474 (2009).

52. Klump, K. L., Keel, P. K., Sisk, C. \& Burt, S. A. Preliminary evidence that estradiol moderates genetic influences on disordered eating attitudes and behaviors during puberty. Psychol. Med. 40, 1745-1753 (2010).

53. Price, A. L. et al. Principal components analysis corrects for stratification in genome-wide association studies. Nat. Genet. 38, 904-909 (2006).

54. Purcell, S. et al. PLINK: a tool set for whole-genome association and population-based linkage analyses. Am. J. Hum. Genet. 81, 559-575 (2007).

55. Wray, N. R. et al. Research review: Polygenic methods and their application to psychiatric traits. J. Child Psychol. Psychiatry. 55, 1068-1087 (2014).

56. Murray, G. K. et al. Could Polygenic Risk Scores Be Useful in Psychiatry?: A Review. JAMA psychiatry (2020) doi:10.1001/jamapsychiatry.2020.3042.

57. Levine, M. E. et al. A polygenic risk score associated with measures of depressive symptoms among older adults. Biodemography Soc. Biol. 60, 199-211 (2014).

58. Zhang, J.-P. et al. Schizophrenia Polygenic Risk Score as a Predictor of Antipsychotic Efficacy in First-Episode Psychosis. Am. J. Psychiatry 176, 21-28 (2019). 
59. Hübel, C. et al. Binge-eating disorder, anorexia nervosa, and constitutional thinness differ in their associations with anthropometric and psychiatric polygenic scores. medRxiv 2020.03.24.20042648 (2020) doi:10.1101/2020.03.24.20042648.

60. Vandereycken, W. Denial of illness in anorexia nervosa-a conceptual review: part 1 diagnostic significance and assessment. Eur. Eat. Disord. Rev. 14, 341-351 (2006).

61. Vandereycken, W. Denial of illness in anorexia nervosa-a conceptual review: part 2 different forms and meanings. Eur. Eat. Disord. Rev. 14, 352-368 (2006).

62. Duncan, L. et al. Significant Locus and Metabolic Genetic Correlations Revealed in Genome-Wide Association Study of Anorexia Nervosa. Am. J. Psychiatry 174, 850-858 (2017).

63. Nagata, J. M. et al. Genetic risk, body mass index, and weight control behaviors: Unlocking the triad. Int. J. Eat. Disord. 52, 825-833 (2019).

64. Abdulkadir, M. et al. Polygenic Score for Body Mass Index Is Associated with Disordered Eating in a General Population Cohort. J. Clin. Med. 9, 1187 (2020).

65. Ursini, G. et al. Convergence of placenta biology and genetic risk for schizophrenia. Nat. Med. 24, 792-801 (2018).

66. Vassos, E. et al. Lack of Support for the Genes by Early Environment Interaction Hypothesis in the Pathogenesis of Schizophrenia. Schizophr. Bull. (2021) doi:10.1093/schbul/sbab052.

67. Kim, Y., Zerwas, S., Trace, S. E. \& Sullivan, P. F. Schizophrenia genetics: where next? Schizophr. Bull. 37, 456-463 (2011).

68. Lewis, C. M. \& Vassos, E. Prospects for using risk scores in polygenic medicine. Genome Med. 9, 96 (2017).

69. Wonderlich, S. A., Bulik, C. M., Schmidt, U., Steiger, H. \& Hoek, H. W. Severe and enduring anorexia nervosa: Update and observations about the current clinical reality. Int. J. Eat. Disord. 53, 1303-1312 (2020).

70. Kaye, W. H. \& Bulik, C. M. A crisis in care: The treatment of anorexia nervosa in the US. JAMA Psychiatry.

\section{Figures}


A
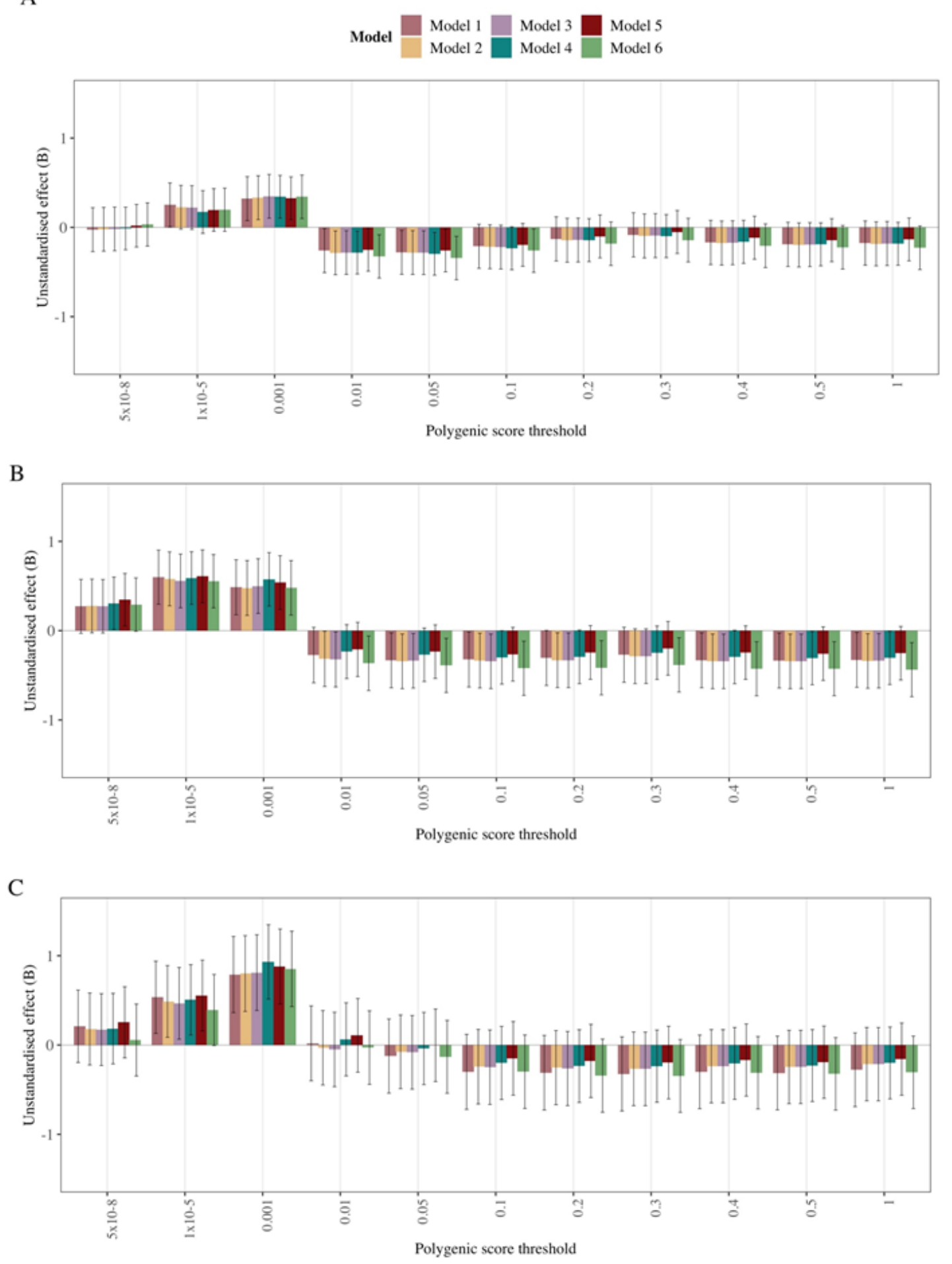

\section{Figure 1}

Anorexia nervosa polygenic score (PGS) associated with disorder severity measured as clinical impairment assessment (CIA) total score. Bars represent beta estimates +/- standard errors. Panel $A$ is based on 2,843 individuals with anorexia nervosa and additional eating disorder diagnoses during their lifetime. Panel B is based on 1,839 individuals with any clinically ascertained anorexia nervosa diagnosis. Panel C is based on the 976 individuals with a clinically ascertained anorexia nervosa restricting subtype. 
The estimates in model 4 are adjusted for covariates included in the main model: Principal components 1-10, treatment region, age, sex, follow-up time and eating disorder diagnosis when including individuals with any eating disorder diagnosis $(\mathrm{N}=2,843)$.

A
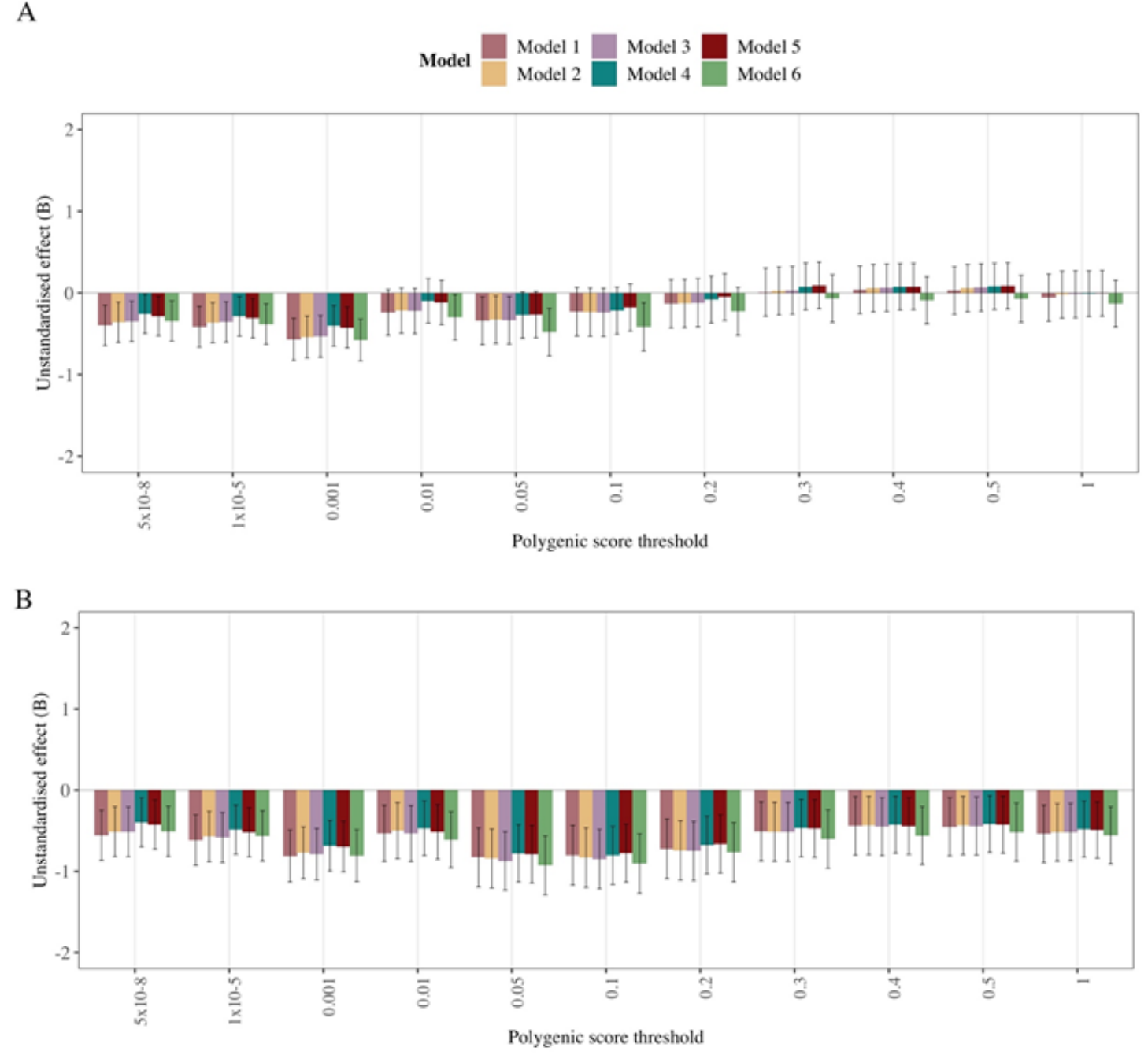

$\mathrm{C}$

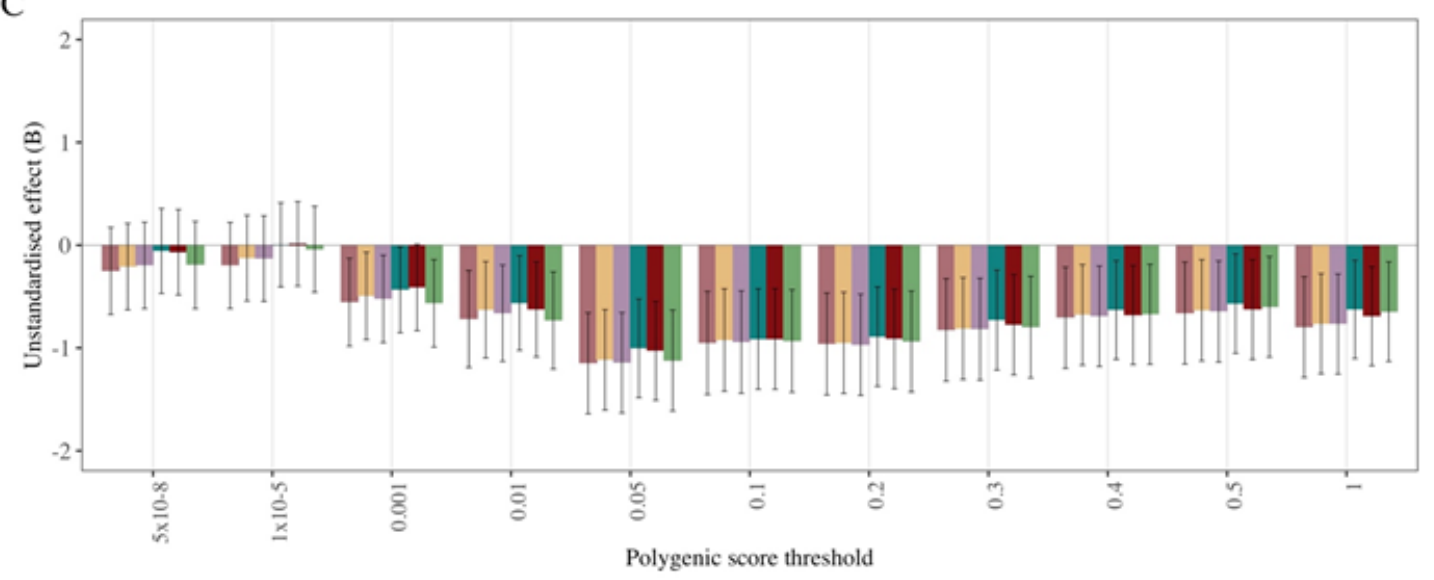

Figure 2

Schizophrenia polygenic score (PGS) associated with disorder severity measured as clinical impairment assessment (CIA) total score. Bars represent beta estimates +/- standard errors. Panel A is based on 
2,843 individuals with anorexia nervosa and additional eating disorder diagnoses during their lifetime. Panel $B$ is based on 1,839 individuals with any clinically ascertained anorexia nervosa diagnosis. Panel $C$ is based on 976 individuals with a clinically ascertained anorexia nervosa restricting subtype. The estimates in model 4 are adjusted for covariates included in the main model: Principal components 1-10, treatment region, age, sex, follow-up time and eating disorder diagnosis when including individuals with any eating disorder diagnosis $(\mathrm{N}=2,843)$.

A
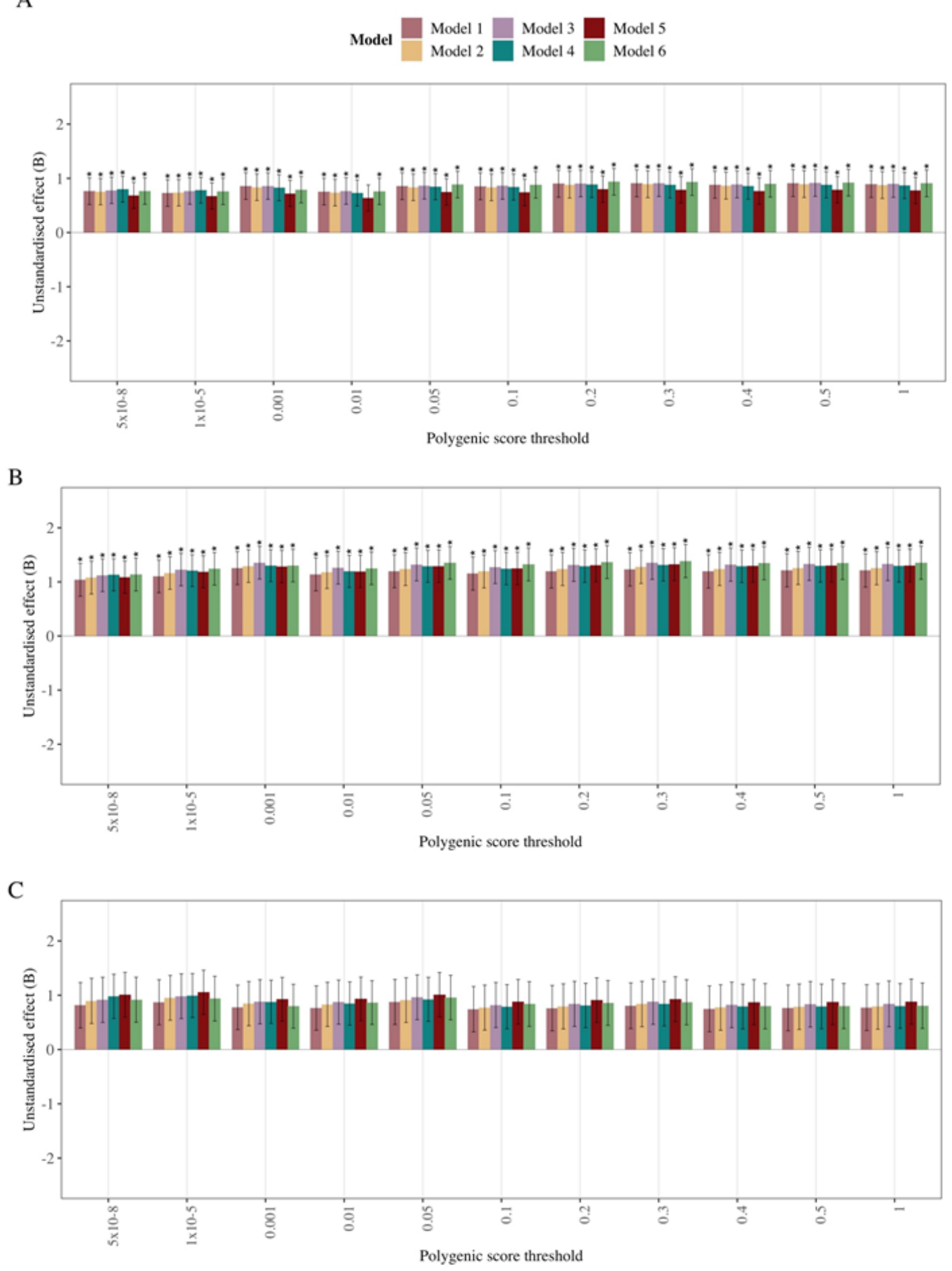

Figure 3 
Body mass index (BMI) polygenic score (PGS) associated with disorder severity measured as clinical impairment assessment (CIA) total score. Bars represent beta estimates +/- standard errors. Panel A is based on 2,843 individuals with anorexia nervosa and additional eating disorder diagnoses during their lifetime. Panel $B$ is based on 1,839 individuals with any clinically ascertained anorexia nervosa diagnosis. Panel $C$ is based on 976 individuals with a clinically ascertained anorexia nervosa restricting subtype. The estimates in model 4 are adjusted for covariates included in the main model: Principal components 1-10, treatment region, age, sex, follow-up time and eating disorder diagnosis when including individuals with any eating disorder diagnosis $(\mathrm{N}=2,843)$. The asterisk $\left(^{*}\right)$ denotes statistically significant results (a $=0.008)$. 
Figure 4A-I
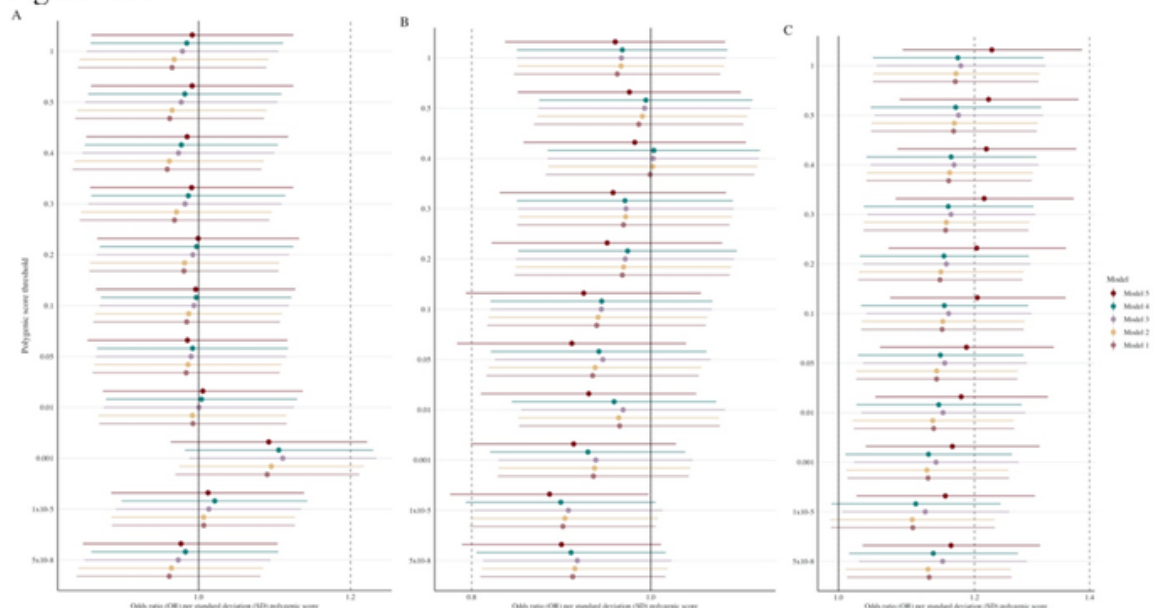

Figure 4D-F
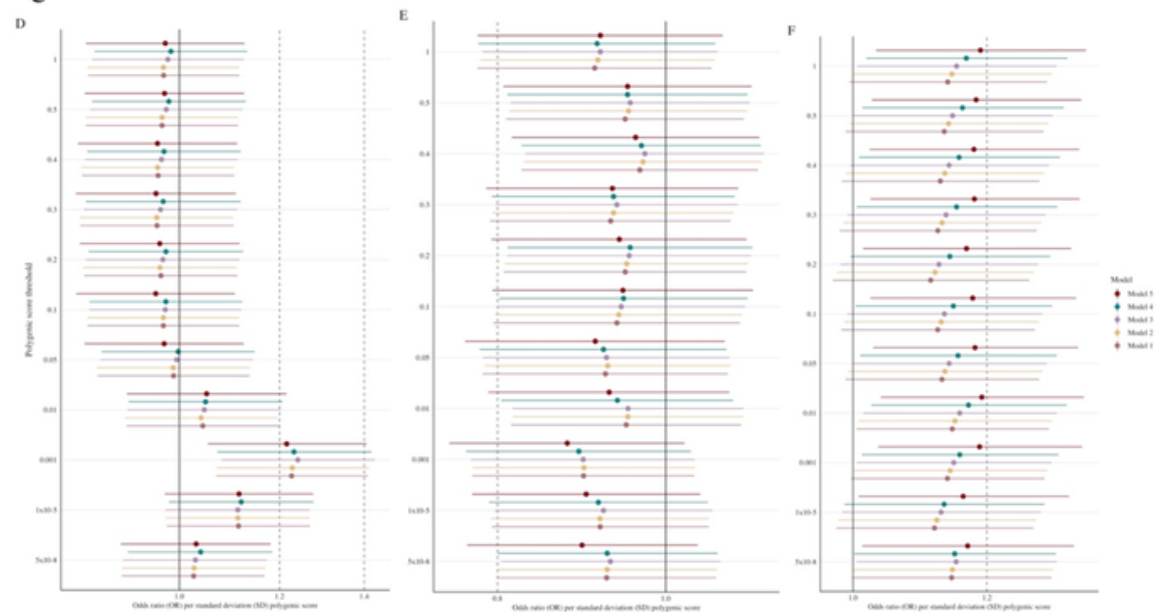

Figure 4G-I
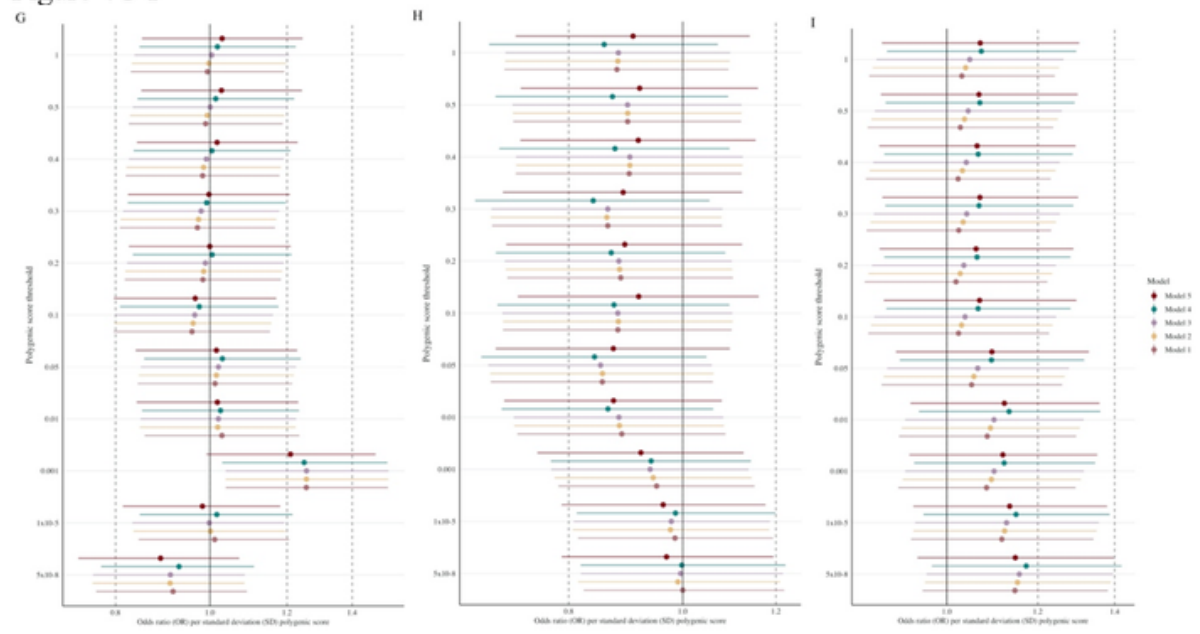

\section{Figure 4}

4A-C. Polygenic scores (PGSs) associated with a severe and enduring eating disorder (SEED). Figure 4A-C is based on 1,334 individuals with anorexia nervosa and additional eating disorder diagnoses during their lifetime ( $\mathrm{n}$ SEED $=528, \mathrm{n}$ non-SEED $=806$ ). Figure 4A shows the association with the anorexia nervosa PGS, Figure 4B shows the association with the schizophrenia PGS, and Figure 4C shows association with the BMI PGS. The estimates in model 3 are adjusted for covariates included in the main model: Principal 
components 1-10, treatment region, age, sex and eating disorder diagnosis when including individuals with any eating disorder diagnosis. Dots represent odds ratios (ORs) and error bars $95 \%$ confidence intervals.

\section{Supplementary Files}

This is a list of supplementary files associated with this preprint. Click to download.

- 24Sep2021.supplementary.tables.xlsx

- 24Sep2021SupplementalMethodsFigures.docx 